\title{
A Review of the Relationship between Obesity and
}

\section{Circulating Vitamin D Levels: Strength and Directionality}

\begin{abstract}
Raouf MA*
International Management-Health Services and Research Institute, Indianapolis, IN, USA
\end{abstract}

*Corresponding author: Raouf M Afifi, MD, ITM-HS\& Research Institute, 13835

\section{Review Article \\ Volume 1 Issue 2}

Received Date: October 23, 2017

Published Date: November 04, 2017

Azalea Circle 201 Tampa Fl. 33613, USA, E-mail: raoufafifi43@gmail.com

\section{Abstract}

Obesity is the chronic disease of the time of a myriad of associated dietetic and behavioral factors. Vitamin D deficiency (VDD) has also been linked to obesity. This work reflects an interest in studying the relationship between obesity and vitamin D deficiency in the community. A thorough review to examine available data addressing the underlying mechanisms; correlates and causal relationship between the two conditions would be carried out. Understanding the association trends between obesity and VDD states helps improve policies and suggest recommendations for the control and prevention of obesity -related VDD complex, especially among risk populations.

Keywords: Obesity; Vitamin D deficiency; Mechanisms; Causality

\section{Introduction}

This review is undertaken in a climate of a global concern about the burden of obesity and its connection to a wide range of chronic health derangements, including musculoskeletal disorders and the group of cardio metabolic diseases encompassing obesity, diabetes mellitus (DM), hypertension (HTN), and cardiac disease [1]. Vitamin D deficiency may be involved in mechanisms associated with fat cells accumulation, adding more complexity to the chronic obesity-VDD health problem [2]. Together with the epidemic widespread of unhealthy diet, physical inactivity, reliance on technology indoors lifestyle to save effort and time, both VDD and obesity, if not controlled will be a threat to the public's health. A multidisciplinary approach, including both traditional and innovative interventions to control obesity and lifestyle-related chronic metabolic diseases is critical. Such approaches include, but are not limited to, advocating medical advances; health education and policy control. Further, prevention at all levels of healthcare, e.g., community screening programs, early detection of VDD in high-risk patients, and improved access to quality health services can be most costeffective. Moreover, the devastating implications of advanced stages of obesity on cardiovascular health and risk of mortality justify evidence-based surgical intervention (bariatric surgery) to control those risks. This work aims to presenting an updated review to identify bases of the relationship between obesity and VDD, addressing the underlying biophysiological mechanisms and the correlates, and studying the causal directionality of this relationship. Understanding the association trends between the two diseases helps improve policies and suggest recommendations for the control and prevention of obesity -related VDD complex, particularly among risk populations.

\section{Methodology}

Study design: A literature review to study the relationship between obesity, of variable degrees, and VDD would be carried out.

Literature review approach: Meticulous search considering a systematic hierarchy of evidence and utilizing quality and ranked resources to explore and critique facts and arguments about the VDD- obesity pathway would be contemplated. Our thorough search contemplated tracking the development of knowledge about obesity and VDD either separately or in 
relationship to each other. Advanced search would continue until most updated information and evidence based findings have been revealed.

Data sources: An extensive literature search was conducted on the relationship between obesity of variable degrees and body fat distribution and VDD states to identify published articles or reports till to date has been carried out. Ranked search engines, such as PubMed, Ovid Medline, Cochrane library, and Google Scholar, have been used. (A manual search of reference lists of original studies was also searched).

Study selection process: If the selected studies were published in a scientific journal, the latter should have at least an ISSN number (with or without online ISSN number). Only studies published in English and where full manuscripts were reviewed. A special attention of the sampling techniques to assure maximum validity and representativeness of the studied populations were given. Further, studies published in abstract form were excluded.

Specific areas of review included, but were not limited to, evaluating the epidemiology of obesity and VDD Conditions, associated risks, mechanisms and path physiological factors involving circulating vitamin D level as a correlate of fat distribution pattern in obese patients. (A hint on the clinical, diagnostic and remedial strategies for obesity-VDD track is also given).

Revealed Information from this study can provide theoretical database to improve our state of knowledge about the combined obesity and VDD problem in today's populations towards minimizing its impact upon the patients' quality of life (qol) and community's health.

\section{Literature review}

\section{Prevalence and Distribution Trends}

Obesity is a significant health problem world-wide of some variability in its prevalence and burden, being worse in developing countries [3]. Ever since the change in the world's dietary and physical activity (PA) patterns, the world populations are growing fatter. Most populations and ages, including childhood-age groups, are now affected. In 2014, more than 1.9 billion adults 18 and older were overweight, including 600 million who were obese [4]. These figures represent $39 \%$ and $13 \%$ of adult population, respectively.

\section{Obesity Identification and Assessment}

Obesity is generally evaluated by means of body mass index (BMI) (an indicator of body fat calculated by dividing a person's weight in kilograms by their height in meters squared). Based on BMI principle, the World Health Organization (WHO) defines increased body weight in terms of BMI $25 \mathrm{~kg} / \mathrm{m}^{2}=$ overweight and BMI $30 \mathrm{~kg} / \mathrm{m}^{2}=$ obese [4]. Commonly used anthropometric weight and body mass techniques include BMI, waist circumference (WC) and waist-hip ratio (WHR) [5]. These measures are used as diagnostic and follow indices of obesity in a variety of clinical and research settings. In people with a BMI $<35$, WC may be used in addition to BMI. Because of their high correlation, from the statistical perspective, BMI and WC are unlikely to yield different answers [6]. Governments are often condemned of being insufficiently capable and oriented of the most major risks to health affecting the world's population. Among the recommendations are improvements in long-term diet, increased PA levels, early education, and interventions which may include behavior and lifestyle.

Mechanisms, precipitating factors, and conditions in linkage with obesity: Several factors have been incriminated in the development of obesity, including genetic factors, energy imbalance, fat cell theory (such that an increased proliferation of fat cells and adipose tissues during infancy and childhood predisposes to adulthood obesity) [7], behavioral and psychological factors, including irresistance to eating, eating while watching television have an apparent role in weight gain [8]. There is a multitude of health problems associated with obesity. The heightened adipose tissue mass together with the metabolic changes of obesity can induce serious health problems, increasing the risk for many chronic ailments [9], e.g., hypertension (HTN) and cardiovascular disease (CVD) [1]. Obese persons have more than double risk to have hypercholesterolemia. Type 2 diabetes mellitus (T2DM is also known with its strong positive relationship with obesity, and peculiarly potentiating the state of insulin resistance [10]. Most these complications can be prevented by weight loss, even by modest weight loss.

\section{Vitamin D levels and Obesity}

Recently, overweight and obesity have been shown to be related to low vitamin D status. Around 840 million people worldwide suffer from vitamin D deficiency [11] which may result from limited exposure to sunlight, long-term wearing of covering clothes, use of sunscreen, age, low consumption of food containing ergocalciferol, and malabsorption syndrome [12]. Vitamin D receptor (VDR) and the $1 \alpha$-hydroxylase enzyme, which catalyzes the conversion of calcidiol [25-hydroxyvitamin D, or $25(\mathrm{OH}) \mathrm{D}]$ to calcitriol [1, 25-dihydroxyvitamin D or activated vitamin $\mathrm{D}[1,25(\mathrm{OH}) 2 \mathrm{D}]$, were found in more than 40 human cell types indicating its potential role in the regulation of numerous metabolic processes [12]. According to rather recent data, there may be a 
connection between vitamin D levels and cardio metabolic diseases collated in the "metabolic syndrome [13]." The latter involves a combination of central obesity, hyperglycemia, HTN, and atherogenic dyslipidemia [14]. Although the mechanisms are still unclear, VDD is associated with a greater risk of these pathological conditions [15]. On the other hand, an increased body fat and obesity may well be associated with low circulating $25(\mathrm{OH})$ D levels [13].

\section{Causal Relationship and Directionality of the Obesity-Vitamin D Relationship}

Researchers earlier were influenced by the hypothesis that obesity was risk factor for developing decreased circulating vitamin $D$, but not vice versa [13]. It has been consistently observed that increased body fat (BF) content; a prime manifestation of obesity, and obesity both predispose to lowered $25(\mathrm{OH}) \mathrm{D}$ levels, in a straightforward suggestion of a temporal relationship between high and morbidly high BMI and reduced vitamin D levels and its impaired metabolic pathways. Going one step further, the hypothesis that maintained vitamin D level could be protective against obesity and increased fat mass in risky people has emerged [2]. This direction of the relationship between chronic VDD and obesity could also have a role in the development of the metabolic syndrome as one whole entity, or any of its individual aggregates [12]. As such and despite the consistent link between vitamin D and obesity, the exact explanations of causality needs to be further scrutinized. In the presence of an widespread prevalence of obesity among most population subsets and a similar impaired vitamin D levels trend, in conjunction with widespread diet scarce in vitamins, alongside with indoors and inactive lifestyle, undertaking large-scale experiments with large number of lean controls vs. the obese group, observe for variability in vitamin D levels and record accurate diet history over years of study period is most needed but rather difficult. In a longitudinal study by Leblanc et al. (2012) on 9,704 US cohort of women aged $\geq 65$, higher vitamin D levels were consistently associated with lower weight gain [4,16]; so low vitamin D may predispose to fat accumulation, and vice versa. More recently, a study led by the Institute of Child Health, University College of London (ICH-UCL), genetic markers were used as instrumental variables in bidirectional Mendelian randomization analysis. On such basis of a bi-directional genetic approach that limited confounding, it has been suggested that a higher BMI leads to lower serum 25(OH)D levels, while any effects of lower 25(OH)D increasing BMI are likely to be small. However, it has also been emphasized by the same study that population level interventions to reduce BMI are expected to decrease the prevalence of VDD [11].
Yet, in the context of lack of vitamin D supplementation epidemic already linked both to low bone mineral density and obesity, a study to examine the relationship between serum 25(OH)D, anthropometric measures, $\mathrm{BF}$, and bone structure at the time of peak bone mass was carried out.[16] Ninety post-pubertal Californian young women aged 16-22y. Anthropometric measures, serum 250HD radioimmunoassay (RIA) values, and computed tomography (CT) and dual-energy x-ray absorptiometry (DEXA) values of BF and bone structure were measured. Approximately $59 \%$ of subjects were $25(\mathrm{OH})$ D insufficient $(\leq 29 \mathrm{ng} / \mathrm{ml})$, and $41 \%$ were sufficient $(\geq 30$ $\mathrm{ng} / \mathrm{ml})$. Strong negative relationships were present between serum $25(\mathrm{OH}) \mathrm{D}$ and CT measures of visceral adipose tissue (VAT) and subcutaneous fat (SCF), and DEXA values of BF. In addition, weight, body mass, and imaging measures of adiposity at all sites were significantly lower in women with normal serum $25(\mathrm{OH}) \mathrm{D}$ concentrations than those with insufficient levels. (No relationship was observed between 25(OH) D concentration and DEXA at any site; but unexpectedly, there was a positive correlation between 25(OHD) levels and height). Therefore, it could be concluded that that VDD was associated with increased BF (but not changes in peak bone mass).In the older age population, a Kaiser Permanente study looked at more than 4,600 women aged 65 and older for a four and one-half year period. Women with low circulating vitamin D levels significantly gained about two pounds more than those with adequate levels of the vitamin. The longer VDD the greater likelihood to build-up more weight [16].

And in a similar context of a growing belief that calcium and vitamin $\mathrm{D}(\mathrm{CaD})$ may play a role in the regulation of abdominal fat mass in older adults, a study investigated the effect of CaD-fortified orange juice (OJ) on weight loss and VAT reduction among overweight and obese adults (mean $40.0 \pm 12.9 y$ ). Two parallel, double-blinded, placebo-controlled trials were conducted with regular vs. reduced-energy (lite) 0J. For each 16-wk trial, 171 participants were randomly assigned to 1 of 2 groups. The average weight loss $(\approx 2.45 \mathrm{~kg})$ did not differ between groups. However, in the regular OJ trial, the reduction of VAT was significantly greater among the CaD group than in the control group. In the lite OJ trial, the reduction of VAT was significantly greater in the $\mathrm{CaD}$ group than in controls (after controlling for baseline VAT). The effect of calcium/vitamin D on VAT remained highly significant when the results of the 2 trials were combined. As in the younger age group before, it may well be suggested that vitamin $\mathrm{D}$ and/or calcium supplementation contributes to a beneficial reduction of VAT [17]. This proves that vitamin D plays an important role in keeping our body in shape [18]. 
The pleiotropic role of vitamin D where many organs and a multitude of metabolic processes are influenced by serum $25(\mathrm{OH})$ D has been clearly addressed. For instance, the risks of CVD, DM, HTN and dyslipidemia have been linked with VDD [18-24]. Adipokines are involved in a number of biological and physiological processes, too $[22,25]$. They play an important role in body homeostasis influencing food intake, glucose and lipid metabolism, vascular remodeling, and insulin action $[22,23,26]$.

Consistently, the importance of fatty tissue is visible in obesity as well as in malnutrition [22]. A fat-rich diet contributes to the accumulation of adipose tissue, which leads to insulin resistance. Additionally, hypertrophy of adipocytes is connected with increased synthesis and secretion of pro-inflammatory mediators such as: TNF$\alpha$, IL-6 (interleukin-6), and IL-1 [22,27]. It also leads to increased concentrations of circulating cytokines and promotes adipose tissue inflammation, characterized by macrophages infiltration. While an increased secretion of TNF- $\alpha$ and IL- 6 cytokinesis involved in the pathogenesis of obesity, and a reduction of adiponectin also does so $[18,22,27]$.

Leptin secretion in the context of obesity and VDD states: Particularly leptin is a circulating hormone produced primarily by adipose tissue and its biosynthesis is operated by leptin gene (OB) located on the 7 th chromosome. Leptin synthesis is stimulated by insulin, glucocorticosteroids, TNF- $\alpha$ and estrogens, and inhibited by free fatty acids (FFAs) and growth hormone [22]. Once secreted from adipocytes into the bloodstream, leptin is a principal Adipokines meant to regulate food intake and metabolism [22,26]. The concentration of circulating leptin increases with fat mass and decreases with caloric restriction and body mass reduction [22]. Leptin secretion also follows a circadian rhythm (the highest concentration is observed between 22:00 and 3:00, which could explain the reduction of food intake at this time). Therefore, leptin is a "satiety hormone" where it inhibits the synthesis of Neuro peptide-Y and hence suppresses appetite. Leptin works by sending a signal to brain cells and stimulates the individual's satiety sensation, leading to cessation of eating [28]. Vitamin D, in turn, supports such leptin signaling so that in VD-deficient persons, interrupted lept in signaling leads to disrupted fullness sensation mechanism and this causes overeating and subsequent obesity. In addition, leptin has a pleiotropic action in organism physiology and behavior regulation. Nonetheless, the clinical significance of the connections between leptin and vitamin $D$ remains to be ascertained [21].

In their attempt to answer the question whether vitamin D repletion supports weight loss and changes obesity-related biomarkers, Xiao and collaborates (2014) recruited 218 overweight/obese women (50-75 $\mathrm{y}$ of age) with serum $25(\mathrm{OH}) \mathrm{D} \geq 10 \mathrm{ng} / \mathrm{mL}$ but $<32$ $\mathrm{ng} / \mathrm{mL}$ [29]. The study cohort was randomly assigned to either weight loss +2000 IU oral vitamin D3/d or weight loss + daily placebo. Mean 12-month changes in weight, body composition, serum insulin, and $25(\mathrm{OH}) \mathrm{D}$ were compared between groups (intent-to-treat) by using generalized estimating equations. The mean change in 25(OH) D in the vitamin D3 arm among the intervention group (a positive change) was significantly higher than that of the placebo group (a negative change). Changes in weight, $\mathrm{BMI}, \mathrm{WC}, \% \mathrm{BF}$, trunk fat, and insulin, all were not significantly different between the two groups. However, compared with women who achieved 25(OH)D $<32 \mathrm{ng} / \mathrm{mL}$, women randomly assigned to vitamin D supplement who became replete (i.e., $25(\mathrm{OH}) \mathrm{D} \geq 32 \mathrm{ng} / \mathrm{mL})$ significantly lost more weight: $-8.8(-11.1,-6.9 \mathrm{CI})$, compared with $-5.6(-7.2$, $-5.0 \mathrm{CI}) \mathrm{kg}$; WC: $-6.6(-9.3,-4.3)$, compared with -2.5 $(-4.6,-2.0) \mathrm{cm}$; and percentage $\mathrm{BF}:-4.7(-6.1,-3.5)$, compared with $-2.6 \quad(-3.9,-2.2)$; $(\mathrm{p}<0.0 \mathrm{f}$ all comparisons). The study provides that vitamin D3 supplementation during weight loss did not increase weight loss or associated factors compared with placebo; however, women who became replete experienced greater improvements.

\section{Vitamin D Biophysiology}

Vitamin D is synthesized in the skin (after UV light exposure) or consumed, and then is stored until needed. When serum concentration of vitamin $\mathrm{D}$ is below normal, both intestinal calcium and phosphorus absorption decrease [15]. Calcitriol stimulates intestinal calcium absorption by interacting with vitamin D receptors in the small intestine [30]. Therefore, calcitriol's major biologic activity on bone is indirect: to promote passive mineralization of collagen matrix (osteoid) by maintaining extracellular calcium and phosphorus in a supersaturated state. Vitamin D is 25hydroxylated in the liver in a largely unregulated step and activated to calcitriol by $25(\mathrm{OH}) \mathrm{D}-1-\alpha$ hydroxylase in the kidney (tightly regulated) [31]. In the physiological state of calcium deficiency, PTH levels rise resulting in renal 1-hydroxylation of $25(\mathrm{OH}) \mathrm{D}$ and increased production of $1,25(\mathrm{OH}) 2 \mathrm{D}$. The main dietary sources of vitamin D (D2) are vitamin D fortified milk, margarine and cereal, although fish, liver and egg yolks are good but lesser sources. Sun exposure (ultraviolet ray-B) (UVB) is the predominant source of vitamin D (D3) via photo-conversion of vitamin D precursors to pre-vitamin D3 in skin. According to the National Institute of Medicine, persons are at risk of vitamin D deficiency at serum 25(OH) D concentrations $<30$ $\mathrm{nmol} / \mathrm{L}(<12 \mathrm{ng} / \mathrm{mL})$. Some are potentially at risk for inadequacy at levels ranging from $30-50 \mathrm{nmol} / \mathrm{L}$ (12- 
$20 \mathrm{ng} / \mathrm{mL}$ ). Practically all people are sufficient at levels $\geq 50 \mathrm{nmol} / \mathrm{L}(\geq 20 \mathrm{ng} / \mathrm{mL})$; and concentrations $>125$ $\mathrm{nmol} / \mathrm{L}(>50 \mathrm{ng} / \mathrm{mL})$ are associated with potential adverse effects.

\section{Clinical Importance and Symptomatology}

Given its fat-soluble nature (fat acts as a "sink" by collecting it), obese patients are more prone to developing VDD symptoms than the non-obese [32]. Vitamin D impairment has been incriminated in bone aches, in combination with fatigue, which often ends up with being misdiagnosed as having fibromyalgia or chronic fatigue syndrome [33]. The clinical consequences of VDD include osteomalacia, increased susceptibility to fragility fractures, bone pain, and muscle weakness. Vitamin D deficiency should be suspected in patients who receive little or no direct sunlight, vitamin D deficient diets, and fat malabsorption syndromes (e.g., Crohn's disease and intestinal bypass surgery) [34].

\section{Laboratory Methods and Biochemical Analyses}

Tests involving obesity-VDD symptoms include serum vitamin D level (25[OH [D); plasma parathyroid hormone (PTH) level; serum calcium, and fasting plasma glucose (FPG) levels. Vitamin D deficiency may be defined according to reference values as serum concentrations of $25(\mathrm{OH}) \quad \mathrm{D}<10 \mathrm{ng} / \mathrm{ml}$. Vitamin D insufficiency may be defined as 10 to $<19.9 \mathrm{ng} / \mathrm{ml}$. A serum concentration of $25(\mathrm{OH}) \quad \mathrm{D} \geq 20 \mathrm{ng} / \mathrm{ml}$ is considered as adequate vitamin D level. [For the sake of metabolic syndrome diagnosis, DM is defined according to WHO standard of diagnosis of glucose intolerance is fasting plasma glucose (FPG) $\geq 126 \mathrm{mg} / \mathrm{dl}$; high total cholesterol (HC) is defined according to the Adult Treatment Panel III (ATP III) guidelines as HC $\geq 240$ $\mathrm{mg} / \mathrm{dl}]$ [35].

\section{Treatment and Prevention of Obesity-VDD Conditions}

While there is no generally agreed upon treatment for VDD, a commonly used strategy is vitamin D 40,000 units weekly for 7 weeks $(280,000$ units) and then followed by 4,000 units a day for 10 weeks $(280,000$ units), followed by 800-2000 units maintenance dose daily [36]. A target point recommended for VDD is $>30$ $\mathrm{ng} / \mathrm{ml}$. Bottom-line, controlling the underlying co morbidities is the cornerstone of VDD treatment. Factoring the peculiar relationship between $25(\mathrm{OH}) \mathrm{D}$, on the one hand, and BMI and fat mass setup on the other hand, adequacy and stabilization of either component entails stabilization of the other. Obesity, in turn is one of the most difficult and discouraging problems to treat, and long-term success rates remain low. Specialized weight loss programs are essential measures for weight reduction. Surgical intervention (e.g., bariatric surgery) in order to control severe obesity remains an effective method of weight loss, and often yields partial or complete resolution of multiple obesity-related co morbidities. The morbidly obese and obese with BMI $\geq 35$ and or more comorbid, e.g., T2DM, HTN, sleep apnea, osteoarthritis, lipid abnormalities or CVD are candidates. Dietary supplementation (vitamins $\mathrm{B} 12$ and $\mathrm{D}, \mathrm{Zn}, \mathrm{Fe}$ ) after surgery is usually indicated.

\section{Conclusion}

Obesity and vitamin D deficiency are two chronic and nutritional health problems inflicting a persistently growing number of today's populations of all age ranges and demographic traits. Both diseases have a wide range of metabolic and systemic complications, some of which are triggered by the two conditions equivocally. For instance, both obesity and VDD can be risk for metabolic syndrome components, e.g., CVD, dm, HTN and dyslipidemic symptoms. In fact, both obesity and VDD often emerge from a commonly unfavorable epidemiological and behavioral background of physical inactivity, high caloric diet; inadequate vitamin supply and long hours of indoors staying. Further, the affinity of vitamin $\mathrm{D}$ to adipose tissue so that a large adipose tissue mass tends to retain an accumulating concentrations of $25(\mathrm{OH}) \mathrm{D}$ has been significantly observed both in clinical setting and by laboratory measurement, e.g., reduced serum 25(OH)D levels were associated by enhanced CT signals of visceral and subcutaneous fat, and likewise, BMI imaging measures of adiposity in the body become lower with 25(OH)D levels have been normalized, compared to insufficient vitamin D level states. In turn, a reduced circulating vitamin D negatively influences target organs, e.g., causing impaired bone mineralization and metabolic and systemic derangements, as above. Now that vitamin D was found to be playing an important role in regulating abdominal fat mass, the longer VDD the more proneness to building-up more weight, and a vicious circle of obesity- VDD keeps rolling. To date, it is widely agreed upon that obesity and VDD are commonly see in association with each other. First, it had been found that obesity can be risk for VDD, especially in high risk people, such as old women, morbidly obese, multiple chronic comorbid conditions, being home-bound and less physically active. For instance, it is quite common that an increased body fat, as in obese people is associated with low circulating $25(\mathrm{OH}) \mathrm{D}$ levels. By time, investigators started looking at the obesity- VDD question from the standpoint of vitamin $\mathrm{D}$ as a precursor not only an outcome. It has been noted that adequate vitamin D supplementation can effectively assist in preventing advancement to obesity, and the hypothesis that VDD itself can be risk for obesity and 
that maintained vitamin D level could be protective against increased fat mass now gains an increasing momentum. The burden of obesity and VDD upon the populations' health and national economies is tremendous, particularly in the presence of a countless number of complications and comorbidities in close linkage with these conditions. Therefore and early detection through widespread community screening programs in parallel with health education for risk people would be most cost-effective. A remarkable success has been achieved in the research of fat tissue volume and distribution manifested by obesity and impaired circulating vitamin D level states in recent years, including the underlying physiological and hormonal background. However, investment in further experimentation on mechanisms, correlates, and causal relationship between vitamin $\mathrm{D}$ and body fat distribution pathologies should be contemplated. Examining the strength of evidence of the influence of VDD upon developing clinical obesity and complications in different clinical, demographic and community settings is still warranted.

\section{References}

1. American Heart Association (AHA) (2014) Obesity Information.

2. Leblanc ES, Rizzo JH, Pedula KL, Ensrud KE, Cauley J, et al. (2012) Associations between 25hydroxyvitamin D and weight gain in elderly women. Journal of Women Health 21(10): 10661073.

3. World Health organization (WHO) (2017) Global Health Observatory (GHO) data. Overweight and obesity.

4. Ahmad N, Adam SA, Nawi AM, Hassan MR, Ghazi HF (2016) Abdominal Obesity Indicators: Waist Circumference or Waist-to-hip Ratio in Malaysian Adults Population. Int J Prev Med 7: 82.

5. Tanamas SK, Ng WL, Backholer K, Hodge A, Zimmet PZ, et al. (2016) Quantifying the proportion of deaths due to body mass index- and waist circumference-defined obesity.Obesity (Silver Spring) 24(3): 735-742.

6. Qin X, Zhang Y, Cai Y, He M, Sun L, et al. (2012) Prevalence of obesity, abdominal obesity and associated factors in hypertensive adults aged 4575 years. Clin Nutr 32(3): 361-367.

7. Lim SL, Bruce AS (2015) Prospect theory and body mass: characterizing psychological parameters for weight-related risk attitudes and weight-gain aversion. Front Psychol 6: 330.
8. Lee EB (2011) Obesity, leptin, and Alzheimer's disease. Ann N Y Acad Sci 1243: 15-29.

9. Sanada H, Yokokawa H, Yoneda M, Yatabe J, Sasaki YM, et al. (2012) High body mass index is an important risk factor for the development of type 2 diabetes. Intern Med 51(14): 1821-1826.

10. Vimaleswaran KS, Berry DJ, Lu C, Tikkanen E, Pilz S, et al. (2013) Causal relationship between obesity and vitamin D status: bi-directional Mendelian randomization analysis of multiple cohorts. PLoS Med 10 (2): 1-14. Article e1001383

11. Alam J, Jan SS (2016) Association between vitamin $\mathrm{d}$ deficiency and depressive illness in adult population J Med Sci 24(4): 247-250.

12. McGill AT, Stewart JM, Lithander FE, Strik CM, Poppitt SD (2008) Relationships of low serum vitamin D3 with anthropometry and markers of the metabolic syndrome and diabetes in overweight and obesity. Nutrition Journal 7: 4.

13. Srikanthan K, Feyh A, Visweshwar H, Shapiro JI, Sodhi K (2016) Systematic Review of Metabolic Syndrome Biomarkers: A Panel for Early Detection, Management, and Risk Stratification in the West Virginian Population. Int J Med Sci 13(1): 25-38.

14. Forman JP, Curhan GC, Taylor EN (2008) Plasma 25-hydroxyvitamin $\mathrm{D}$ levels and risk of incident hypertension among young women. Hypertension 52(5): 828-832.

15. Kremer R, Campbell PP, Reinhardt T, Gilsanz V (2009) Vitamin D Status and Its Relationship to Body Fat, Final Height, and Peak Bone Mass in Young Women. The Journal of Clinical Endocrinology \& Metabolism 94(1): 67-73.

16. Rosenblum JL, Castro VM, Moore CE, Kaplan LM (2012) Calcium and vitamin D supplementation is associated with decreased abdominal visceral adipose tissue in overweight and obese adults. Am J ClinNutr 95(1): 101-108.

17. Ding C, Gao D, Wilding J, Trayhurn P, Bing C (2012) Vitamin D signalling in adipose tissue. Br J Nutr 108(11): 1915-1923.

18. Frankiewicz T (2011) Vitamin D supplementationis it only osteo protection? Prz Menopauz 4: 328333.

19. Gannagé-Yared MH, Chedid R, Khalife S, Azzi E, Zoghbi F, et al. (2009) Vitamin D in relation to metabolic risk factors, insulin sensitivity and 


\section{Bioequivalence \& Bioavailability International Journal}

adiponectin in a young Middle-Eastern population. J Endocrinol 160(6): 965-971.

20. Menendez C, Lage M, Peino R, Baldelli R, Concheiro P, et al. (2001) Retinoic acid and vitamin D3 powerfully inhibit in vitro leptin secretion by human adipose tissue. J Endocrinol 170(2): 425431.

21. Skowrońska B, Fichna M, Fichna P (2005) The role of adipose tissue in endocrine system. Endokrynol Otyłość 1: 21-29.

22. Ulutas O, Taskapan H, Taskapan MC, Temel I (2013) Vitamin D deficiency, insulin resistance, serum adipokine, and leptin levels in peritoneal dialysis patients. Int Urol Nephrol 45(3): 879-884.

23. Vaidya A, Pojoga L, Underwood PC, Forman JP, Hopkins PN, et al. (2011) The association of plasma resistin with dietary sodium manipulation, the renin-angiotensin-aldosterone system, and 25hydroxyvitamin D3 in human hypertension. Clin Endocrinol (Oxf) 74(3): 294-299.

24. Małecki MT (2006) Obesity--insulin resistance-type 2 diabetes mellitus. Kardiol Pol 64(10): S561566.

25. de Luis DA, Soto GD, Conde R, Izaola O, de la Fuente B (2012) Relation of leptin and adiponectin with cardiovascular risk factors, intact parathormone, and vitamin $D$ levels in patients with primary hyperparathyroidism. J Clin Lab Anal 26(5): 398402.

26. Lira FS, Rosa JC, Cunha CA, Ribeiro EB, do Nascimento CO, et al. (2011) Supplementing alphatocopherol (vitamin E) and vitamin D3 in high fat diet decrease IL-6 production in murine epididymal adipose tissue and 3T3-L1 adipocytes following LPS stimulation. Lipids Health Dis 10: 37.
27. Casanueva FF, Diéguez C (1999) Neuroendocrine regulation and actions of leptin. Frontiers in Neuroendocrinology 20(4): 317-363.

28. Xiao L, Imayama I, Duggan C, Wang CY, Korde L, et al. (2014) Vitamin D3 supplementation during weight loss: a double-blind randomized controlled trial. Am J Clin Nutr 99(5): 1015-1025.

29. Denio A (2012) The International Society for Clinical Densitometry, Vitamin D Deficiency: The Silent Epidemic of the Elderly.

30. Takahashi N, Udagawa N, Suda T (2014) Vitamin D endocrine system and osteoclasts. Bone Key Reports 3: 495.

31. National Institute of Health (NIH) (2016) Vitamin D. Fact sheet for health professionals.

32. Hossein-nezhad A, Holick MF (2013) Vitamin D for Health: A Global Perspective Mayo Clinic Proceedings 88(7): 720-755.

33. Ham M, Longhi MS, Lahiff C, Cheifetz A, Robson S, et al. (2014) Vitamin D Levels in Adults with Crohn's Disease Are Responsive to Disease Activity and Treatment. Inflamm Bowel Dis 20(5): 856-860.

34. Shils ME, Shike M (2006) Modern Nutrition in Health and Disease, 1st ed.; Lippincott Williams \& Wilkins: Philadelphia, PA, USA.

35. New Therapies Subgroup (2016) Treatment of Vitamin D Deficiency and Insufficiency in Adults.

36. American Society for Metabolic and Bariatric Surgery. Who is a Candidate for Bariatric Surgery. 